\title{
A proto brown dwarf candidate in Taurus
}

\author{
D. Barrado ${ }^{1}$, M. Morales-Calderón ${ }^{1,2}$, A. Palau ${ }^{1}$, A. Bayo ${ }^{1}$, I. de Gregorio-Monsalvo ${ }^{3}$, C. Eiroa $^{4}$, N. Huélamo ${ }^{1}$, \\ H. Bouy ${ }^{5,6}$, O. Morata ${ }^{7,8}$, and L. Schmidtobreick ${ }^{3}$
}

\author{
1 LAEX-CAB, depto. Astrofísica (INTA-CSIC), PO Box 78, 28691 Villanueva de la Cañada (Madrid), Spain \\ e-mail: barrado@cab.inta-csic.es \\ 2 Spitzer Science Center, California Institute of Technology, Pasadena, CA 91125, USA \\ European Southern Observatory, Alonso de Córdova 3107, Vitacura, Santiago, Chile \\ ${ }^{4}$ Depto. Física Teórica, Facultad de Ciencias, Universidad Autónoma de Madrid, 28049 Madrid, Spain \\ 5 Instituto de Astrofísica de Canarias, Av. Vía Láctea s/n, 38200 La Laguna, Spain \\ ${ }^{6}$ European Space Agency (ESAC), PO Box 78, 28691 Villanueva de la Cañada (Madrid), Spain \\ 7 Academia Sinica, Institute of Astronomy and Astrophysics, PO Box 23-141, Taipei 106, Taiwan \\ 8 Department of Earth Sciences, National Taiwan Normal University, 88 Sec.4, Ting Chou Rd., Taipei 116, Taiwan
}

Received 4 April 2009 / Accepted 22 September 2009

\section{ABSTRACT}

\begin{abstract}
Aims. We search for brown dwarfs at the Class 0/I evolutionary stage, or proto brown dwarfs.
Methods. We present a multi wavelength study, ranging from optical at $0.8 \mu \mathrm{m}$ to radio wavelengths at $6 \mathrm{~cm}$, of a cool, very faint, and red multiple object, SSTB213 J041757, detected by Spitzer toward the Barnard 213 dark cloud, in Taurus.

Results. The SED of SSTB213 J041757 displays a clear excess at long wavelengths resembling that of a Class I object. The mid-IR source has two possible counterparts, A and B, in the near-IR and optical images, and the $350 \mu \mathrm{m}$ observations detect clear extended emission, presumably from an envelope around the two sources. The position of A \& B in the $(I c-J)$ versus $(J-[3.6])$ colour-colour diagram is consistent with them being Galactic sources and not extragalactic contaminants. A proper-motion study confirms this result for A, while it is inconclusive for B. The temperature and mass of the two possible central objects, according to COND evolutionary models, range between $1550-1750 \mathrm{~K}$ and 3-4 $M_{\text {Jupiter }}$, and 950-1300 K and 1-2 $M_{\text {Jupiter }}$, for A and B, respectively. The integrated SED provides bolometric temperatures and luminosities of $280 \mathrm{~K}$ and $0.0034 L_{\odot}$, assuming that the emission at wavelengths $>5 \mu \mathrm{m}$ is associated with component $\mathrm{A}$, and $150 \mathrm{~K}$ and $0.0033 L_{\odot}$, assuming that the emission at wavelengths $>5 \mu \mathrm{m}$ is associated with component B, which would imply the SSTB213 J041757 object has a luminosity well below the luminosity of other very low luminosity objects discovered up to date.

Conclusions. With these characteristics, SSTB213 J041757 seems to be a promising, and perhaps double, proto brown dwarf candidate.
\end{abstract}

Key words. circumstellar matter - stars: formation - stars: low-mass, brown dwarfs - stars: pre-main sequence - infrared: stars

\section{Introduction}

Since the discovery of the first brown dwarfs in the field and in stellar associations (Nakajima et al. 1995; Rebolo et al. 1995), a large number of substellar objects $\left(<0.072 M_{\odot}\right)$ have been identified and studied. However, their formation mechanism remains unclear: do they form like a star, from collapse and fragmentation of the initial molecular cloud (Padoan \& Nordlund 2004), since we do not see any change in their properties when we compare them with low-mass stars? Or do they form like planets, from the circumstellar material present around more massive stars, and are they later expelled by gravitational interactions (Reipurth \& Clarke 2001; Bate et al. 2002)? Or, are they protostellar cores that have lost their envelopes because of the action of strong winds from nearby, massive stars (Whitworth \& Zinnecker 2004)? Since stars and brown dwarfs evolve very rapidly during the first million years, the answer to the formation mechanism (or mechanisms) must be in the study of their properties when they are deeply embedded in the natal cloud, a stage referred to as "proto brown dwarf". This would correspond to the Class 0/I stage in the classical evolutionary scheme of young stellar objects (e.g., Lada 1987; André 1994). If we find proto brown dwarfs surrounded by substantial disks and envelopes, similar to those observed in the first stages of low-mass protostars (André et al. 1993), this would provide direct support for the in situ formation of brown dwarfs.

However, no brown dwarf has been unambiguously discovered in the Class 0/I stage. The youngest known bona fide brown dwarfs have ages around $\simeq 1$ Myr (White et al. 1999; Mohanty et al. 2004; Luhman et al. 2005) and are Classical TTauri analogs (Barrado y Navascués \& Martín 2003), characterised by moderate infrared excesses originating in a circumstellar disk, the source of the accreted material falling onto the central object, and no envelope (in the scheme of Lada 1987, a Class II object). Phan-Bao et al. (2008) also discovered a Class II brown dwarf with a faint molecular outflow.

By using data collected by the Spitzer Space Telescope (Werner et al. 2004), several research groups have found socalled very low luminosity objects (VeLLOs), which are objects embedded within dense cores of internal luminosities (protostellar+circumstellar) less than $0.1 L_{\odot}$ (Di Francesco et al. 2007), and are the most likely candidate brown dwarfs in the Class 0/I phase, although their true substellar nature is not clear (e.g., Lee 2007). Only a handful of VeLLOs has been studied in detail (for instance, André et al. 1999; Young et al. 2004; Kauffmann et al. 2005, 2008; Lee et al. 2009), the least luminous having a bolometric luminosity of $\sim 0.1 L_{\odot}$ (Lee et al. 2009). Therefore, we 
developed a strategy to search systematically for proto brown dwarfs (Morales-Calderón et al. 2009, in prep.).

\section{A multi wavelength approach to the search for a proto brown dwarf}

\subsection{Identification of proto brown dwarf candidates based on Spitzer data}

By using Spitzer data from the IPAC database, we identified several faint, red objects in the dark cloud Barnard 213, which belongs to the Taurus-Auriga complex, located at $140 \mathrm{pc}$. These objects were classified as Class 0/I candidate members (see additional details in Morales-Calderón et al. 2009, in prep.). Here, we conduct a comprehensive study of one of them, namely SSTB213 J041757.75+274105.5 (hereafter SSTB213 J041757).

The IRAC data were collected during February 21, 2005. IRAC imaging (Fazio et al. 2004) was performed in mapping mode with individual exposures of $12 \mathrm{~s}$ (corresponding to $10.4 \mathrm{~s}$ exposure times) at each map step and with no dither. Each bandpass map covers an area of $\sim 1.14$ degrees. The IRAC mosaics do not exactly cover the same FOV in all bands, providing an area of $\sim 1.12$ degrees centered on 04:19:19.45 +27:50:38.3, imaged in the four bandpasses. All of the following study refers to objects in that area. We also queried the archive for MIPS data of B213 and analyzed data corresponding to February 28, 2005 (MIPS, AORID:11229696). The MIPS map of the area was performed with fast scan-rate mode and 12 legs separated by 302 arcsec in the cross-scan direction. The total effective integration time per point on the sky at $24 \mu \mathrm{m}$ for most points in the map was $15 \mathrm{~s}$, and the mosaic covered an area of $1 \times 1.4$ degrees centered around 04:19:47.96 +27:49:08.53. Therefore, there is an area slice of $\sim 8 \times 76$ arcmin west of the region with IRAC but not MIPS data. We initially used the Spitzer Science Center pipeline mosaics when extracting the photometry. However, we verified the positions of our selected candidates to see whether there were visible artifacts in the mosaics. The Spitzer photometry was extracted within the IRAF environment.

SSTB213 J041757 occupies the same location as Class 0/I sources in the IRAC colour-colour diagram (see Fig. 1a), although it is very close to the edge of the area reserved to Class II objects (classical TTauri stars), following Allen et al. (2004). In the diagram, we distinguish between previously known members of Taurus (Luhman et al. 2006), displayed as black asterisks, and objects detected in our analysis of the Spitzer public data: solid red circles for Class 0/I, solid blue triangles for Class I/II, green crosses for Class II or CTTs, and solid red squares for Class III or diskless stars (including weak-lined TTauri stars).

Among the Class 0/I candidates in Fig. 1a, we selected those with magnitudes consistent with a substellar nature (following Baraffe et al. 2003). Figure 1b displays a colour-magnitude diagram (symbols as in Fig. 1a), where we have included a $1 \mathrm{Myr}$ isochrone (dusty) from the Lyon group and marked the borderline between stars and brown dwarfs (without taking into account reddening, although note the reddening vector for $A_{V} \sim$ $10 \mathrm{mag}$ ). Since SSTB213 J041757, in addition to other Class I candidate members, is located several magnitudes fainter than this substellar limit, it appears to be an excellent target for additional follow-up. We observed a subsample of these candidates with the IRAM $30 \mathrm{~m}$ Telescope at $1.2 \mathrm{~mm}$ (in on-off mode, in May, Oct., Nov. 2007, and Nov. 2008), and identified SSTB213 J041757 to be possibly the most embedded target among them (we note however that its final flux at $1.2 \mathrm{~mm}$ was detected with a lower significance than $4 \sigma$, thus we adopted
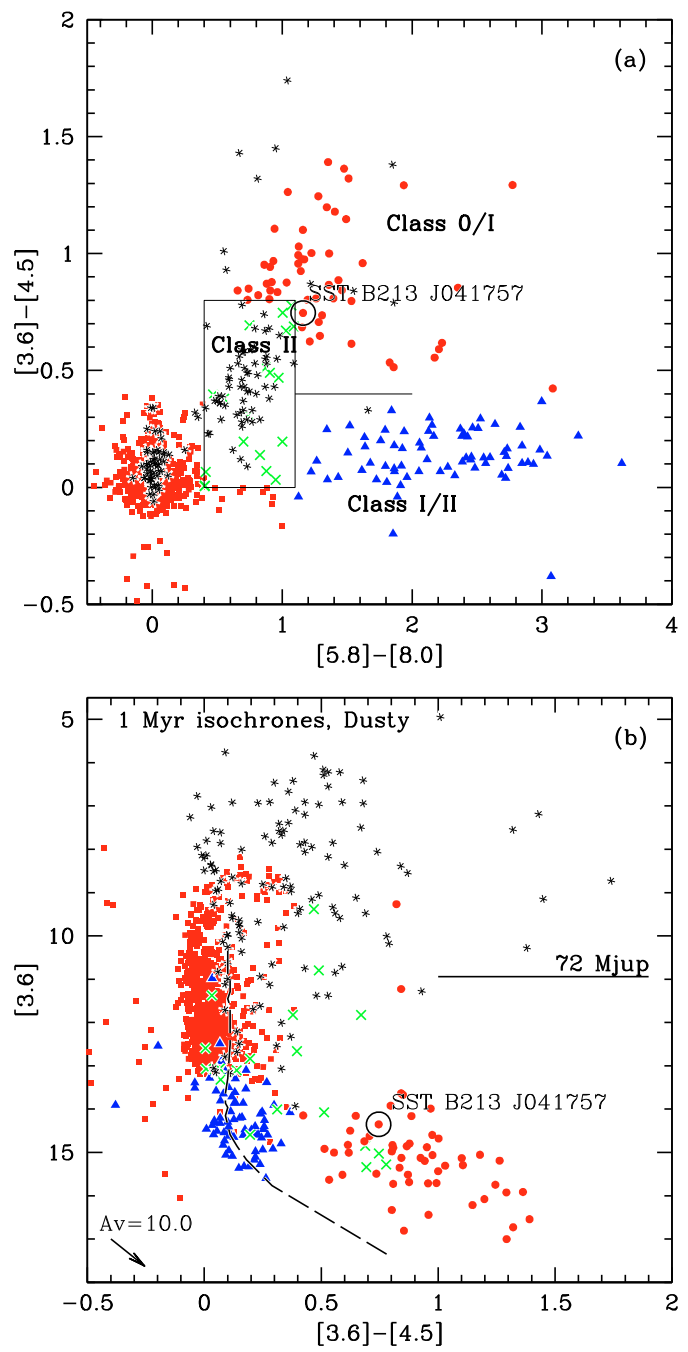

Fig. 1. Spitzer/IRAC colour-colour and colour-magnitude diagrams of sources in the Barnard 213 dark cloud. Small solid squares (red), crosses (green), solid triangles (blue), and solid circles (red) represent Class III, II, I/II and 0/I, respectively. SSTB213 J041757 is identified with a large open circle and a label. The classification scheme follows Allen et al. (2004). The colour-magnitude diagram includes a $1 \mathrm{Myr}$ isochrone dusty - from the Lyon group (Baraffe et al. 2003). A reddening vector is also included. The asterisks represent the location of previously known members of Taurus, with photometry from Luhman et al. (2006)

an upper limit to the flux at this wavelength). Complete details about this campaign and the sample will be provided in MoralesCalderón et al. (2009, in prep.). Figure $1 \mathrm{~b}$ also illustrates that, although a significant number of brown dwarfs have been detected in Taurus, they have moderate infrared excesses, and no proto brown dwarf has been identified in this association or any identification elsewhere is ambiguous. We acknowledge that we are exploring uncharted waters, and that the contamination by extragalactic sources and very extincted stars (mainly background giants) can mimic the properties of a potential substellar member of the cluster (either Class I or II). To avoid these problems and to characterize the properties of our candidate, we carried out exhaustive follow-up observations and analyses of data at different wavelengths, including data retrieved from different public archives. 


\subsection{Follow-up}

We requested several observing runs at even longer, as well as shorter, wavelengths, to be able to construct a complete spectral energy distribution (SED).

\subsubsection{Optical and near-infrared data}

We cross-correlated our Spitzer detections with the 2MASS all sky release (Cutri et al. 2003) using a 2.5 arcsec radius. Since typical Spitzer astrometric accuracy is of order 1 arcsec, such a search radius is enough to be sure that we do not miss counterparts and, since the surface density is not very high in this field, the object identification is generally unambiguous. The limiting magnitudes of the 2MASS catalog are about $J$ (limit) $16.8 \mathrm{mag}, H$ (limit) $\sim 16.1 \mathrm{mag}$, and $K$ s(limit) $\sim 15.3 \mathrm{mag}$.

We obtained deep near-IR imaging in November 4th, 2007 with the Calar Alto $3.5 \mathrm{~m}$ (Almería, Spain) and the Omega2000 camera with Discrectionary Director Time. Omega2000 has a $15.36 \times 15.36$ arcmin of field of view. Each image consists of 30 different $60 \mathrm{~s}$ exposures, with a dithering of 15 arcsec. Therefore, the total exposure time for each filter is $30 \mathrm{~min}$. The seeing was about 1.2 arcsec and the photometric calibration was obtained with stars in the 2MASS catalog. Details about the photometry extraction and the calibration can be found in Barrado y Navascués et al. (2007). These datasets are complete to $J(\mathrm{compl}) \sim 21.00 \mathrm{mag}, H(\mathrm{compl}) \sim 20.00 \mathrm{mag}$, and $K \mathrm{~s}(\mathrm{compl}) \sim 19.50 \mathrm{mag}$, well below the deuterium burning limit at $13 M_{\text {Jupiter }}$ The detection limits are $J$ (limit) $\sim 21.50 \mathrm{mag}$, $H$ (limit) $20.50 \mathrm{mag}$, and $K \mathrm{~s}$ (limit) $20.00 \mathrm{mag}$.

The second campaign was conducted at Paranal (Chile) with ISAAC/VLT, by collecting narrow-band infrared imaging (NB2.13), in January 2009.

We also recovered optical data from the CFHT archive (Dec. 5, 2004. Program 2004BF28, P.I. Dougados). A set of 6 dithered images of $300 \mathrm{~s}$ and $360 \mathrm{~s}$ each were acquired with Megaprime/Megacam in the $i$ and $z$ band, respectively. We retrieved the corresponding photometric catalogues and processed images from the CADC MegaPipe public archive. All the photometric data can be found in Tables 1 and 2. To our surprise, we discovered that SSTB213 J041757, which was first identified with Spitzer at a moderately low angular resolution of $\sim 2$ arcsec, might be a multiple system, since there are three optical and near-infrared sources close to the location of the original IRAC position (see Fig. 2). A PSF-fitting analysis of the IRAC I1 and I images allowed us to extract the individual fluxes of sources A and B, B being the brightest (see Table 1). Since the source $C-2.6$ arcsec east of $A-$ is not detected with IRAC, it is not discussed further.

\subsubsection{Submillimeter and radio observations}

We carried out observations at $350 \mu \mathrm{m}$ and centimetre wavelengths, to confirm and characterize the presence of a dusty envelope and/or ionized gas.

The $350 \mu \mathrm{m}$ observations were conducted at the Caltech Submillimetre Observatory (CSO), in Hawaii (USA), in January 28th, 2008, with the SHARC-II bolometer array. The observations were carried out in imaging mode using a lisajous pattern, which provided a field of view of $3.3 \times 3 ! 2$, and the total observing time was $1.5 \mathrm{~h}$. Typical opacities at $225 \mathrm{GHz}$ were around 0.04 . The rms noise reached was about $14 \mathrm{mJy} \mathrm{beam}^{-1}$, the angular resolution was $10^{\prime \prime}$, and the absolute flux uncertainty was estimated to be $\sim 10 \%$, from a measurement of the calibrator
Table 1. Optical (CFHT/MegaCam), near-IR (CAHA), and IRAC photometry for the resolved sources around SSTB213 J041757.75+274105.5 (A and B).

\begin{tabular}{|c|c|c|}
\hline Filter $(\lambda)$ & $\begin{array}{c}M_{\mathrm{A}}(\mathrm{mag}) \\
\mathrm{RA}=04: 17: 57.71 \\
\mathrm{Dec}=+27: 41: 07.2 \\
(\mathrm{~J} 2000.0)\end{array}$ & $\begin{array}{c}M_{\mathrm{B}}(\mathrm{mag}) \\
\mathrm{RA}=04: 17: 57.75 \\
\operatorname{Dec}=+27: 41: 04.8 \\
(\mathrm{~J} 2000.0)\end{array}$ \\
\hline $\begin{array}{l}i(0.75 \mu \mathrm{m})^{1} \\
z(0.90 \mu \mathrm{m})^{1} \\
J(1.25 \mu \mathrm{m}) \\
H(1.65 \mu \mathrm{m}) \\
K \mathrm{~s}(2.17 \mu \mathrm{m}) \\
{[I 1](3.6 \mu \mathrm{m})^{2}} \\
{[I 2](4.5 \mu \mathrm{m})^{2}}\end{array}$ & $\begin{array}{l}21.05 \pm 0.01 \\
19.99 \pm 0.01 \\
17.50 \pm 0.01 \\
16.41 \pm 0.01 \\
15.95 \pm 0.01 \\
15.57 \pm 0.03 \\
15.10 \pm 0.05\end{array}$ & $\begin{array}{l}22.56 \pm 0.03 \\
21.69 \pm 0.03 \\
19.14 \pm 0.04 \\
18.04 \pm 0.04 \\
16.81 \pm 0.03 \\
14.94 \pm 0.02 \\
14.03 \pm 0.03\end{array}$ \\
\hline
\end{tabular}

${ }^{1}$ in $\mathrm{AB}$ system. ${ }^{2}$ PSF fitting.

Table 2. Additional photometric data, unresolved, for SSTB213 J041757.75+274105.5 A and B.

\begin{tabular}{ccccl}
\hline \hline $\begin{array}{c}\lambda \\
(\mu \mathrm{m})\end{array}$ & $\begin{array}{c}S_{v} \\
(\mathrm{mJy})\end{array}$ & $\begin{array}{c}\sigma \\
(\mathrm{mJy})\end{array}$ & $\begin{array}{c}F W H M \\
\left({ }^{\prime \prime}\right)\end{array}$ & Instrument \\
\hline 5.8 & 0.491 & 0.012 & 1.8 & Spitzer-IRAC \\
8.0 & 0.736 & 0.019 & 1.9 & Spitzer-IRAC \\
24 & 4.55 & 0.07 & 6 & Spitzer-MIPS \\
70 & $<33.6$ & $4 \sigma$ & 18 & Spitzer-MIPS \\
350 & 103 & 14 & 10 & CSO/SHARC \\
1200 & $<2.88$ & $4 \sigma$ & 11 & IRAM30 \\
36000 & $<0.28$ & $4 \sigma$ & 10 & VLA \\
60000 & 0.21 & 0.04 & 16 & VLA \\
\hline
\end{tabular}

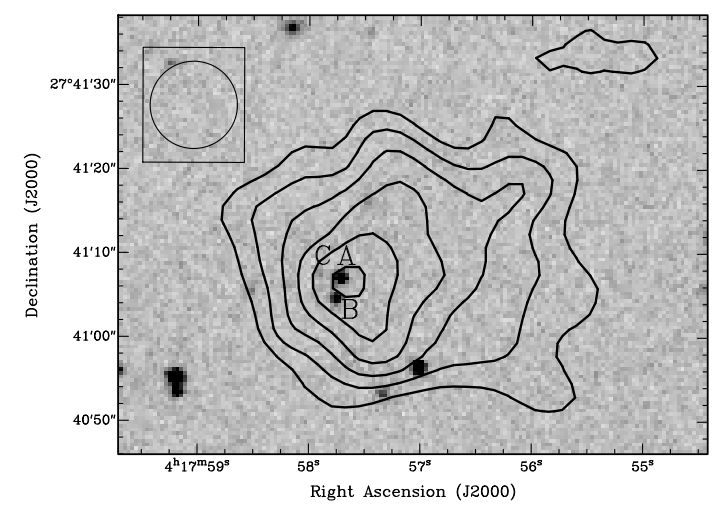

Fig. 2. Grey-scale: CAHA/Omega2000 image taken at $K$ s, with an angular resolution of $0.90^{\prime \prime}$. Contours: CSO $350 \mu \mathrm{m}$ continuum emission with $3,4,5,6,7$, and 8 times the rms of the map, 14 mJy beam $^{-1}$. The beam of the CSO image is shown in the top left corner. Components A, $\mathrm{B}$, and $\mathrm{C}$ in the Omega2000 image are indicated.

HL Tau. The $350 \mu \mathrm{m}$ emission toward SSTB213 J041757 is shown in Fig. 2, and reveals an extended submillimetre source associated with SSTB213 J041757, of about 30 arcsec of size. The peak intensity is $103 \pm 17 \mathrm{mJy}_{\text {beam }}^{-1}$, and the flux density integrated over all the area of emission is $1.1 \pm 0.1 \mathrm{Jy}$.

Finally, we processed radio continuum data at $6 \mathrm{~cm}$ (4.86 GHz) and $3.6 \mathrm{~cm}(8.46 \mathrm{GHz})$ using the Very Large Array (VLA) of the National Radio Astronomy Observatory (NRAO) ${ }^{1}$. The observations were performed on 2008 September 4 with the

1 The National Radio Astronomy Observatory is a facility of the National Science Foundation operated under cooperative agreement by Associated Universities, Inc. 


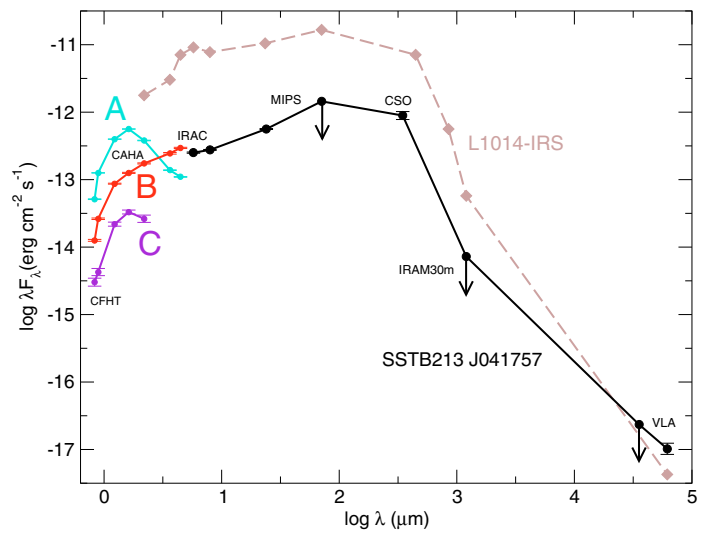

Fig. 3. Spectral energy distribution of SSTB213 J041757, including errorbars, and a comparison with L1014-IRS, a Very Low Luminosity Object (probably an embedded very low-mass young stellar object classified as Class 0/I, Shirley et al. 2007). We differentiate in the diagram between the optical and near-infrared fluxes coming from sources A, B, and $\mathrm{C}$.

array in the D configuration, using the standard VLA continuum mode (4IF, $50 \mathrm{MHz}$ per IF). The time on-source was $\simeq 40 \mathrm{~min}$. The resulting synthesized beam and $\mathrm{rms}$ noise level at $6 \mathrm{~cm}$ were $17.2^{\prime \prime} \times 15.2^{\prime \prime}\left(\mathrm{PA}=77^{\circ}\right)$, and $0.04 \mathrm{mJy}$, respectively. We note that at $3.6 \mathrm{~cm}$ we could only determine an upper limit because of technical problems during the observations. Further details will be given in Palau et al. (2009, in prep.). The $6 \mathrm{~cm}$ observations reveal a compact source at a $5 \sigma$ level that is clearly associated with SSTB213 J041757.

\subsubsection{A complete spectral energy distribution}

Using this wealth of data, we were able to compile a quite complete SED from $0.8 \mu \mathrm{m}$ up to $6 \mathrm{~cm}$. Figure 3 displays the data and differentiates between the $\mathrm{A}, \mathrm{B}$, and $\mathrm{C}$ components of SSTB213 J041757 for optical, and near- and mid-infrared wavelengths, where we were able to derive individual photometry. The diagram also includes a comparison with a VeLLO, namely L1014-IRS, a Class 0/I object (Shirley et al. 2007) discovered by Young et al. (2004). The resemblance between both objects suggests that SSTB213 J041757 is a faint Class 0 or Class I object. We note, however, that our proto brown dwarf candidate is detected at shorter wavelengths, and note the different behavior for A, whose flux peaks at $H$, and $\mathrm{B}$, which has a positive slope in the SED toward longer wavelengths.

\subsection{Extragalactic contaminants}

We carried out several tests to elucidate the nature of the object and try to disregard the possibility of being a background extragalactic source (see more details in Morales-Calderón et al. 2009, in prep.).

The five panels of Fig. 4 contain several colour-magnitude diagrams using the Spitzer photometry (IRAC and MIPS at $24 \mu \mathrm{m})$. All these have been used by different studies (Gutermuth et al. 2008; Harvey et al. 2006; Jørgensen et al. 2006) to discriminate between the population of young stellar and substellar members of nearby stellar associations and contaminants along the line-of-sight, specifically background galaxies. In these diagrams, we included the objects described in Fig. 1 with identical symbols, and added several additional samples with the goal of being able to establish whether
SST B213 J041757 is related to the dark cloud or might share the properties of extragalactic contaminants.

In particular, we included three new samples: i) a selection of the sample listed in Surace et al. (2004), which corresponds to SWIRE/ELAIS. We plot only the objects classified as galaxies, based on Sextrator $(\mathrm{S} / \mathrm{G}$ parameter $\leq 0.05$ in all five criteria). They appear as three-point stars in magenta, which should correspond to normal, resolved galaxies. ii) Data from the GOODS/MUSIC survey (Grazian et al. 2006, which corresponds mainly to active galactic nuclei, are included as upward magenta open triangles. iii) Finally, quasars from the SWIRE study, selected from Hatziminaoglou et al. (2008), are shown as downward open triangle, also in magenta. Since we have individual fluxes for components A and B at 3.6 and $4.5 \mu \mathrm{m}$, both objects were included in panel a (compare with Fig. 1b).

Additional diagrams for diagnostic are displayed in Fig. 5, in particular three colour-colour diagrams (symbols as in Fig. 4).

When combined together, the location of our proto brown dwarf candidate member SST B213 J041757 in all these figures suggests that we are not dealing with an extragalactic contaminant (at least when dealing with the combined photometry of A and B). In some panels, the location of our candidate is in agreement with being a normal galaxy (Figs. 4b, c and 5c). Other panels suggest we might be dealing with an AGN (Figs. 5b, c). Finally, in other cases the location might be compatible with the photometric properties of quasars (Figs. $4 a-c$ and $5 a-c$ ). As a matter of fact, the quasar nature seems to be a very plausible explanation of the nature of SST B213 J041757. However, since some panels (Figs. 4d, e) are in clear disagreement with our object being a quasar, we cannot confirm this possibility. A similar result is obtained in the case of being a galaxy (panels that are in disagreement are Figs. 4a and 5a, b), or an AGN (panels in disagreement are Figs. 4a, b). Thus, we have to conclude that if our object is extragalactic, it must have very peculiar properties.

As an additional test, we compared the position of SSTB213 J041757 in the $(I c-J)$ versus $(J-[3.6])$ diagram (Fig. 6, after Bouy et al. 2009a), and the target is separated well from both quasars (SWIRE survey, Hatziminaoglou et al. 2008), and AGNs (Grazian et al. 2006), in a region where Taurus Class I stars are found. This strongly supports SSTB213 J041757 being either an abnormal extragalactic source or a Galactic source.

Finally, we observed the region in $\mathrm{CO}(1-0)$ with the Kitt Peak telescope (Palau et al. 2009, in prep.) to study the possible outflow emission in the region, and found a faint blueshifted wing (around $1 \mathrm{~km} \mathrm{~s}^{-1}$ blueshifted with respect to the Taurus CO emission), which seems to peak at the position of the target, suggesting that the object is associated with Taurus. In addition, we used the broadest bandpass available to search for possible $\mathrm{CO}$ extragalactic emission between -200 and $200 \mathrm{~km} \mathrm{~s}^{-1}$, and found no significant emission in this velocity range above the $4 \sigma$ level of $0.36 \mathrm{~K}$. Thus, these $\mathrm{CO}$ observations show that some amounts of molecular gas at the velocity of Taurus are associated with the position of the proto brown dwarf candidate. Assuming that this molecular gas is also associated with dust, these $\mathrm{CO}$ observations indicate that the emission at $350 \mu \mathrm{m}$ is most likely associated with the Taurus complex.

As a conclusion, the evidence presented here favour SST B213 J0401757 being Galactic and, hence, a good candidate member of the dark cloud Barnard 213 with a substellar nature.

\subsection{Proper motions}

We used the CFHT 2004 images and the ISAAC 2009 images to search for evidence of the proper motion of the components $\mathrm{A}$ 

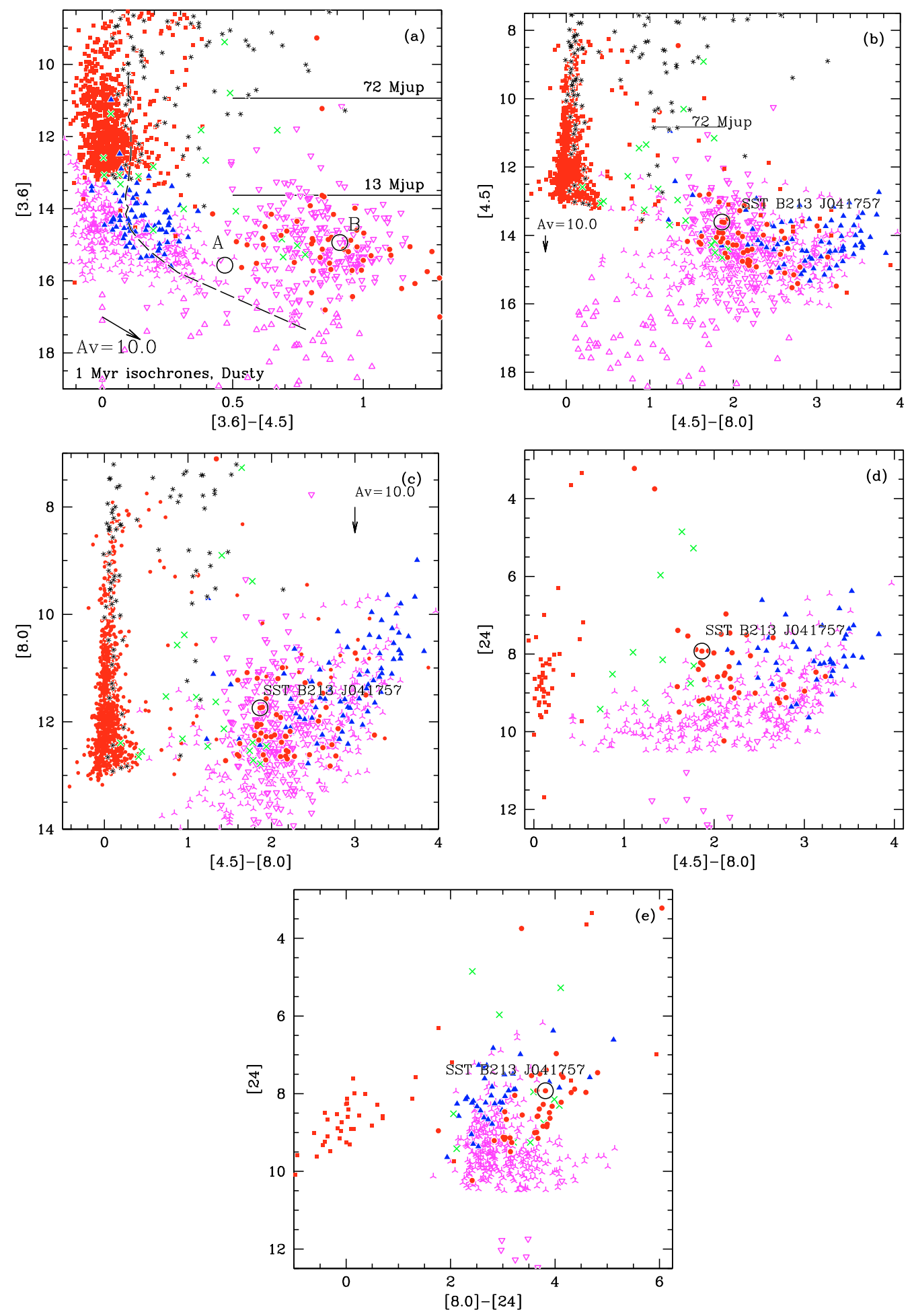

Fig. 4. Spitzer/IRAC colour-magnitude diagrams of sources in the Barnard 213 dark cloud and comparison with extragalactic sources. Small solid squares (red), crosses (green), solid triangles (blue), and solid circles (red) represent Class III, II, I/II, and 0/I, respectively, in Barnard 213. The asterisks represent the location of Taurus previously known members, with photometry coming from Luhman et al. 2006. Regarding the extragalactic sample, up- and down-ward open triangles - magenta - correspond to the samples by Grazian et al. 2006 (GOODS/MUSICS, mainly AGNs) and Hatziminaoglou et al. 2008 (SWIRE/QSO), respectively, whereas three-point stars come from Surace et al. 2004 (SWIRE/ELAIS). SSTB213 J041757 is identified with a big open circle and a label. The first colour-magnitude diagram includes a 1 Myr isochrone - dusty - from the Lyon group (Baraffe et al. 2003). A reddening vector has also been included. Note some extragalactic sources in the samples described here might be outside the limits for the figures (especially in the case of the GOOD/MUSICS sample).

and $\mathrm{B}, \mathrm{C}$ being too faint to derive accurate astrometry. The CFHT images were processed by the Elixir pipeline, which includes image warping and distortion correction. We used deep
ISAAC image of the Omega Cen cluster obtained with the same NB2.13 filter a few weeks after our observations together with HST/ACS images of the same field to calibrate the distortions. 

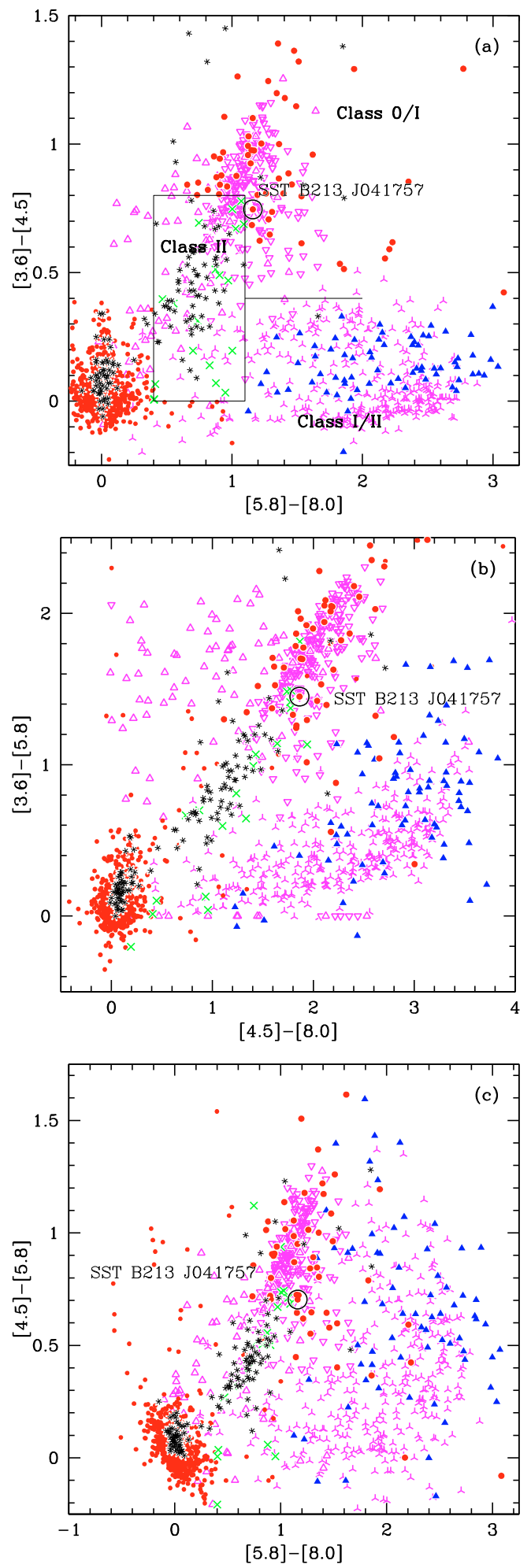

Fig. 5. Spitzer/IRAC colour-colour diagrams of sources in the Barnard 213 dark cloud and comparison with extragalactic sources. Small solid squares (red), crosses (green), solid triangles (blue), and solid circles (red) represent Class III, II, I/II, and 0/I, respectively, in Barnard 213. The asterisks represent the location of Taurus previously known members, with photometry coming from Luhman et al. 2006. Regarding the extragalactic sample, up- and downward open triangles (magenta) correspond to the samples by Grazian et al. 2006 (GOODS/MUSICS, mainly AGNs) and Hatziminaoglou et al. 2008 (SWIRE/QSO), respectively, whereas three-point stars come from Surace et al. (2004) (SWIRE/ELAIS). SSTB213 J041757 is identified with a big open circle and a label.

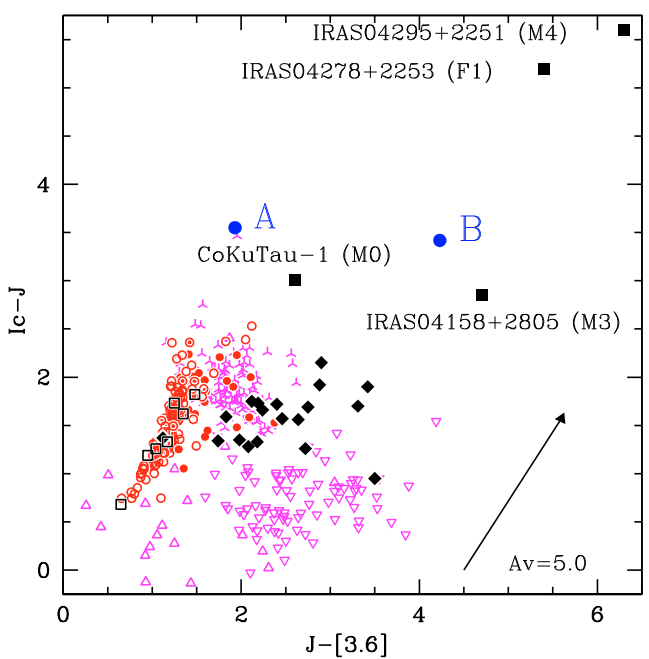

Fig. 6. Optical-infrared colour-colour diagram, after Bouy et al. (2009a). It includes quasars from SWIRE (open magenta triangles, pointing downwards; Hatziminaoglou et al. 2008); AGNs from GOODS-MUSIC (open green triangles, pointing upwards; Grazian et al. 2006); a comparison with the stellar and substellar population of the $5 \mathrm{Myr}$ Collinder 69 cluster (open and solid red circles for Class III and II, respectively; Dolan \& Mathieu 1999, Barrado y Navascués et al. 2007); and Taurus members (open squares, solid diamonds and solid squares (cyan) for Class III, II and I, respectively; Kenyon \& Hartmann 1995, Luhman et al. 2006, and this work).

The relative motion of the two sources were then measured using the method described in Moraux et al. (2001) with the IRAF GEOMAP package. Briefly, fifteen nearby sources were used to compute the ISAAC-to-CFHT transformation matrix. This matrix was then used to compute the 2009 positions of both components A and B in the CFHT 2004 reference frame, and the proper motions were computed by dividing the displacement between the two epochs by the time difference (4.09 yr). Uncertainties were computed by adding quadratically the rms error in the position $(\sim 0.02$ pix $)$ and the rms error in the transformation ( $\sim 0.06$ pix $)$. We report a relative motion of $\mu_{\alpha} \cos (\delta)=$ $-1.5 \pm 4.1 \mathrm{mas} / \mathrm{yr}$ and $\mu_{\delta}=-20.5 \pm 3.5 \mathrm{mas} / \mathrm{yr}$ for component $\mathrm{A}$, and $\mu_{\alpha} \cos (\delta)=2.0 \pm 4.0 \mathrm{mas} / \mathrm{yr}$ and $\mu_{\delta}=-5.5 \pm 3.2 \mathrm{mas} / \mathrm{yr}$ for component B. Taurus members are found to move at $\sim 25 \mathrm{mas} / \mathrm{yr}$ with large dispersion (Bertout \& Geneva 2006, see Fig. 7). While $\mathrm{A}$ is moving at $5.8 \sigma$ with a relative motion consistent with the absolute motion reported for Taurus members, B motion is detected at only $1.7 \sigma$, and does not allow us to draw any firm conclusion about its membership of the association.

The proper motions measured in our CFHT and ISAAC images are relative proper motions, while Ducourant et al. (2005, see Fig. 7) measurements are absolute measurements. A direct comparison is therefore not straightforward. Keeping this limitation in mind, we tentatively derive membership probabilities for A and B using the Ducourant et al. (2005) sample of Taurus members and the method highlighted in Bouy et al. (2009b). We find that components $\mathrm{A}$ and $\mathrm{B}$ have membership probabilities of $99.0 \%$ and $77.8 \%$, respectively. While the membership of A is assessed at a confidence level of $2.57 \sigma$, the membership of B is uncertain at a confidence level of only $1.22 \sigma$.

Thus, while we cannot completely rule out the possibility of source B being extragalactic, the whole dataset seems to point towards both A and B belonging to Taurus, and this is what we assume in the following paragraphs. 


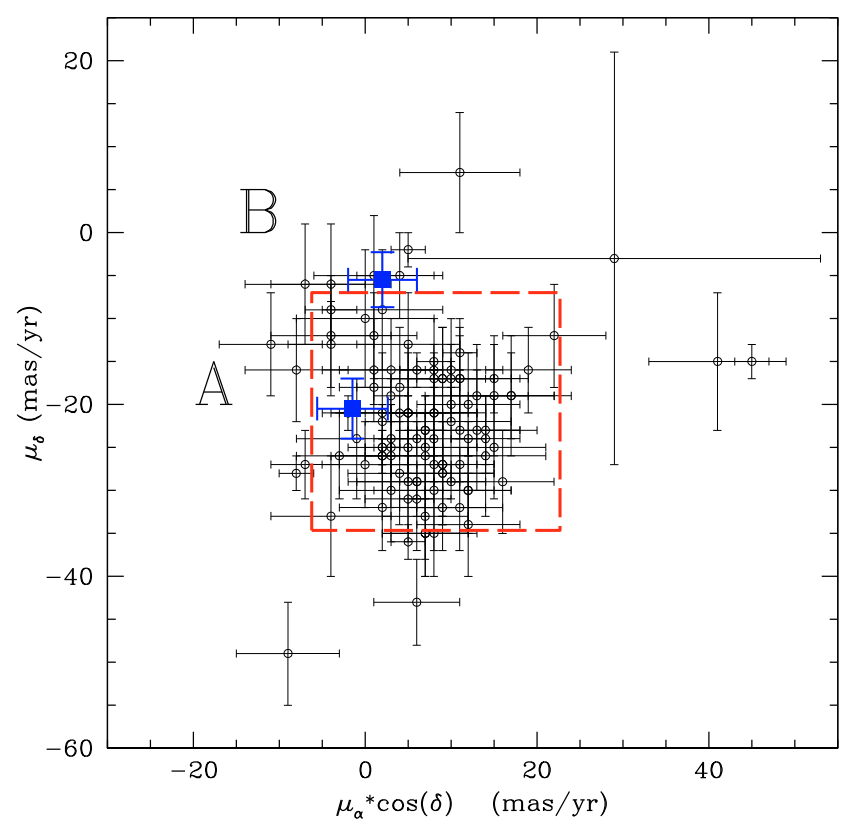

Fig. 7. Proper motions for components A and B of our proto brown dwarf candidate. Taurus members from Ducourant et al. (2005) have been included. The big, long-dashed rectangle (red) delimits the area of bona fide members following Bertout \& Geneva (2006).

\subsection{Properties of SST B213 J041757 as a Taurus candidate member}

Our comprehensive SED (Fig. 3), which includes $4 \sigma$ values as the upper limits, can be discussed in some detail. For the optical and near-IR wavelengths, where we have high angular resolution, source A has a clear maximum in flux at $H$, whereas the flux of B increases towards longer wavelengths. For the unresolved fluxes $(\lambda \geq 5.8 \mu \mathrm{m})$, our object exhibits a smooth increase up to $24 \mu \mathrm{m}$. Taking an upper limit of $4 \sigma$ for the $70 \mu \mathrm{m}$ data-point, the SED would be flat around this wavelength. After the detected emission at $350 \mu \mathrm{m}$, the upper limits are consistent with a decrease in the Rayleigh-Jeans regime of a modified blackbody. Regarding the centimetre range, the detection at $6 \mathrm{~cm}$ is clear, suggesting that the object could be associated with a thermal radio jet or with synchrotron emission. The shape of the SED indicates that we have a possible very young - perhaps double - object with a circumstellar disk, responsible for the emission at shorter wavelengths, which is embedded in a large $(\sim 30$ arcsec, as derived from the CSO image at $350 \mu \mathrm{m})$ and cold $(\sim 12-25 \mathrm{~K})$ envelope, responsible for the submillimetre emission. The mass of the envelope, derived from the integrated flux density at $350 \mu \mathrm{m}$, should be in the range 5-30 $M_{\text {Jupiter }}$, depending on the adopted dust temperature (we used the dust opacity law of Ossenkopf \& Henning 1994; the mass estimate is subject to the uncertainty in the opacity of about a factor of 2). Adopting an envelope mass of $30 M_{\text {Jupiter }}$ and a radius of 15 arcsec, we estimated a visual extinction $A_{v}$ of 4.4, typical of dark clouds (e.g., Myers 1989), and a $\mathrm{H}_{2}$ particle density of $1.2 \times 10^{5} \mathrm{~cm}^{-3}$, characteristic of dense cores, and similar to the density estimated for other VeLLOs (e.g., Shirley et al. 2007). We note that the outer parts of the envelope are most likely gravitationally unbound. By following the expression given in Bontemps et al. (2009) for the virial mass, we estimated the size (in diameter) for which a spherical condensation of $\sim 30 M_{\text {Jupiter }}$ at a kinetic temperature of $\sim 12 \mathrm{~K}$ remains gravitationally bound, and found a size of
300 AU, well within the CSO beam. Finally, it is worth noting that the radial intensity profile of SST B213 J041757 at $350 \mu \mathrm{m}$ is similar to the radial profiles found for VeLLOs ( $\mathrm{Wu}$ et al. 2007). This will be studied in more detail in a subsequent paper (Palau et al. 2009, in prep.).

One can try to classify SST B213 J041757, a substellar candidate, within the classical evolutionary scheme of Class 0-III stages for young stellar objects (e.g., André 1994). The shape of the SED matches the standard criteria for Class 0/I young stellar objects, because it has a positive spectral index between 2 and $25 \mu \mathrm{m}$ in the $\lambda F_{\lambda}$ vs. $\lambda$ diagram (e. g., Wilking et al. 1989; André et al. 2000). In addition, the bolometric temperature (assuming that the emission at wavelengths $>5 \mu \mathrm{m}$ comes from either component $\mathrm{A}$ or component $\mathrm{B}$, see below) falls within the range of Class I sources (from 70 to $650 \mathrm{~K}$, Chen et al. 1995). We remark that in the SED we adopted a flux density at $350 \mu \mathrm{m}$ equal to the peak intensity (instead of integrating over all the emission), because the part of the envelope that is gravitationally bound to the proto brown dwarf most likely falls within the CSO beam, as explained above. Although the bolometric temperature is within the Class I range, SST B213 J041757 may have just recently left the Class 0 phase, because the SED also matches some of the Class 0 criteria. For example, if we compute the ratio $L_{\mathrm{smm}} / L_{\mathrm{bol}}$ (where $L_{\mathrm{smm}}$ is the luminosity longward of $350 \mu \mathrm{m}$ and $L_{\mathrm{bol}}$ is the bolometric luminosity, André et al. 2000) we find that it is $>0.005$, as adopted for Class 0 sources $\left(L_{\mathrm{smm}} / L_{\mathrm{bol}}\right.$ is 0.046 or 0.044 , if we assume that the emission at wavelengths $>5 \mu \mathrm{m}$ is associated with component $\mathrm{B}$, or $\mathrm{A}$, respectively). Other criteria for classifying a source as being in the Class 0 phase is that the SED must peak longward than $100 \mu \mathrm{m}$, as is the case of the SST B213 J041757 SED, and that it must be associated with centrally peaked but extended submillimetre continuum emission (e.g., André et al. 2000). This last criterion for Class 0 sources is what we found toward SST B213 J041757 at $350 \mu \mathrm{m}$, suggesting that the source should be classified in this stage. However, since the SED also matches the Class I criteria, and the object is detected in the optical range, we propose as a conservative approach that SST B213 J041757 is in a phase equivalent to the Class I stage of the star-formation paradigm.

We can try to estimate the nature of sources A and B using their photometry. Assuming that the fluxes at the $J$ and $H$ bands are less affected by the presence of either a disk or an envelope, a COND model by the Lyon group (Baraffe et al. 2003) for an age of $1 \mathrm{Myr}$ and a distance of $140 \mathrm{pc}$ corresponds to masses of 3-4 and 1-2 $M_{\text {Jupiter }}$, radii of 0.20 and $0.17 R_{\odot}$, and $T_{\text {eff }}$ of $1550-1750 \mathrm{~K}$ and $950-1300 \mathrm{~K}$, for sources A and B, respectively. We note that the age of our object might be younger, but this is the lowest value in the theoretical dataset. In any case, all these values are only indicative, since the observed phenomenology is much more complex than the theoretical models and, as stated before, the age is unknown. More appropriate models, including all the phenomenology observed in this source (i.e., disk plus envelope), should be developed to obtain a more accurate characterization of the central object.

The SED also allows us to derive the bolometric temperature and luminosity ( $T_{\mathrm{bol}}$ and $L_{\mathrm{bol}}$ ) of the sources. The estimate of the total luminosity (or rather, an upper limit) can be obtained by a simple integration of our complete SED, assuming a $4 \sigma$ value for the upper limits at $70 \mu \mathrm{m}, 1.2 \mathrm{~mm}$ and $3.6 \mathrm{~cm}$, and a distance of $140 \mathrm{pc}$. In order to do so, we assumed that the emission redwards $5.0 \mu \mathrm{m}$ might arise either from A or B. As a result, we obtain a luminosity of 0.0034 or $0.0033 L_{\odot}$ and a bolometric temperature 


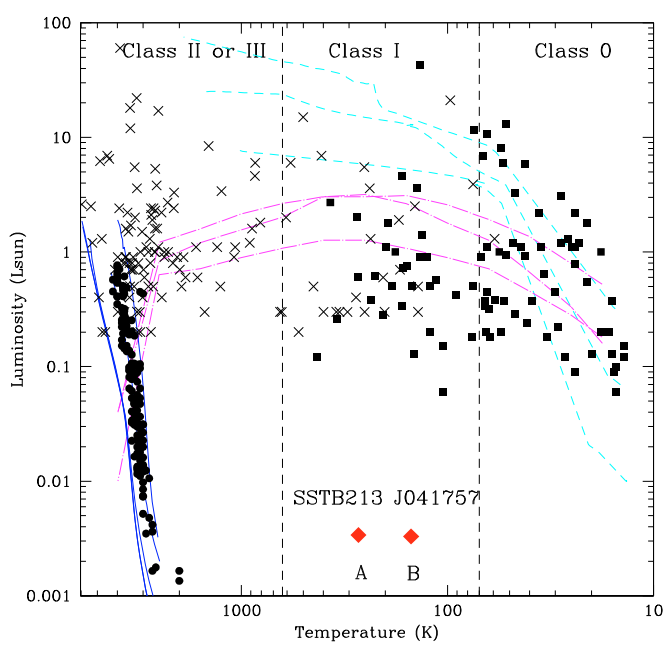

Fig. 8. Bolometric luminosity versus temperature $\left(T_{\text {bol }}\right.$ or $\left.T_{\text {eff }}\right)$ diagram after Young \& Evans (2005) and Dunham et al. (2008). The 50 sources compiled by Dunham et al. (2008), showing some evidence of being embedded low-luminosity sources, are shown as solid, black squares. The long-dashed, magenta lines show the evolutionary tracks of the three models with different masses (in solar masses) considered by Myers et al. (1998). The short-dashed, cyan lines show the evolutionary tracks for three models considered by Young \& Evans. (2005). The crosses correspond to Taurus members and the vertical dashed lines show the Class $0-\mathrm{I}$ and Class I-II $T_{\text {bol }}$ boundaries from Chen et al. (1995). Members from the 5 Myr Collinder 69 cluster are included as solid circles (Barrado y Navascués et al. 2007), as well as isochrones from Baraffe et al. (1998), blue lines. SSTB213 J041757 values of $T_{\text {bol }}$ and $L_{\mathrm{bol}}$ are shown with red diamonds, assuming the extreme cases that all emission at wavelengths $>5 \mu \mathrm{m}$ is associated with either component $\mathrm{A}$, or with $\mathrm{B}$, and assuming that both components are at the Taurus distance. They clearly fall below all the well-characterized VeLLOs known so far.

of 280 or $150 \mathrm{~K}$, for $\mathrm{A}$ and $\mathrm{B}$, respectively ${ }^{2}$. With these values, the mass estimate is $\sim 11 M_{\text {Jupiter }}$ for both of them.

The comparison of the location of the proto brown dwarf candidate in a $L_{\text {bol }}$ versus $T_{\text {bol }}$ diagram (Young et al. 2005, see Fig. 8) shows that our IRAC source, regardless of which component, A or B, accounts for most of the emission at wavelengths $>5 \mu \mathrm{m}$, if associated with Taurus, is different to anything else found so far. For comparison, 50 young sources from Dunham et al. (2008) are shown as solid, black squares. They show evidence of being embedded, low-luminosity sources and have enough photometry to compile their SEDs. The shortdashed cyan lines show the evolutionary tracks of 3 theoretical models of Young \& Evans (2005), which differ in their initial envelope mass. The long-dashed magenta lines show the evolutionary tracks for the models of Myers et al. (1998), which differ in both their initial envelope mass and their final stellar mass. Our IRAC source, marked with red diamonds for the extreme cases that all emission at wavelengths $>5 \mu \mathrm{m}$ is associated with component $\mathrm{A}$ or $\mathrm{B}$, shows the lowest luminosity in the whole sample and appears in the area corresponding to

${ }^{2} T_{\text {bol }}$ and $L_{\mathrm{bol}}$ were obtained by using a flux density at $350 \mu \mathrm{m}$ in the
$\mathrm{SED}$, which is equal to the peak intensity at $350 \mu \mathrm{m}$. If we used the flux
density resulting from integrating the emission over all the envelope,
we would obtain a bolometric luminosity of $0.014 L_{\odot}$ (which is still less
luminous than the known VeLLOs) and a bolometric temperature of 74
or $44 \mathrm{~K}$, for A and B, respectively. However, since the emission outside
the CSO beam is most likely gravitationally unbound to the proto brown
dwarf, we adopt the $T_{\text {bol }}$ and $L_{\text {bol }}$ derived from the SED with the $350 \mu \mathrm{m}$
flux density equal to the $350 \mu \mathrm{m}$ peak intensity, as given in the main text.
Class I objects, in agreement with our classification based on Spitzer/IRAC photometry.

\section{Conclusions}

Following a multi wavelength approach, from the optical to centimetre wavelengths, we have identified a promising - maybe double - proto brown dwarf candidate in the B1213 dark cloud.

1. We have found that the bolometric temperature of SSTB213 J041757 is 150-280 K, whereas the central objects have an approximate effective temperature of 950-1750 K, and that it is embedded in an extended, cold envelope at $\sim 12-25 \mathrm{~K}$ of $5-30 M_{\text {Jupiter }}$.

2. If the source is located at $140 \mathrm{pc}$, its bolometric luminosity is $\sim 0.003 L_{\odot}$.

3 . If the partially extended submillimetre source is really associated with a proto brown dwarf, our observations strongly suggest that the object was not formed by means of the ejection scenario, but rather in a similar way to low-mass stars.

As a secondary result, we present the location of different types of galactic and extragalactic sources in different optical, and near- and mid-infrared colour-colour and color-magnitude diagrams, which might be helpful in establishing the nature of candidate members discovered in other star-forming regions.

Acknowledgements. We deeply thank Calar Alto and IRAM Observatories for allocation of director's discretionary time to this programme. We are deeply grateful to A. Kovacks, S. Leon, R. Zylka and C. Vlahakis for assistance in the reduction of the CSO and IRAM data. The paper is also based on observations obtained with MegaPrime/MegaCam, a joint project of CFHT and CEA/DAPNIA, at the Canada-France-Hawaii Telescope (CFHT) which is operated by the National Research Council (NRC) of Canada, the Institute National des Sciences de l'Univers of the Centre National de la Recherche Scientifique of France, and the University of Hawaii. This research used the facilities of the Canadian Astronomy Data Centre operated by the National Research Council of Canada with the support of the Canadian Space Agency. We do appreciate the discussions with A. Alonso-Herrero, and P. Pérez González, and the contributions from C. Young, and M. Dunham. We are grateful to the anonymous referee for very detailed and useful discussions which have led to the improving the paper. This research has been funded by Spanish grants ESP2007-65475C02-02, AYA2008-06189-C03, AYA2008-1727, CSD2006-00070 and PRICITS-0505/ESP/0361.

\section{References}

Allen, L. E., Calvet, N., D'Alessio, P., et al. 2004, ApJS, 154, 363 André, P. 1994, The cold universe, Proceedings of the 28th Rencontre de Moriond, Les Arcs, France, ed. T. Montmerle, Ch. J. Lada, I. F. Mirabel, \& J. Tran Thanh Van (Gif-sur-Yvette, France: Éditions Frontières), 179 André, P., Ward-Thompson, D., \& Barsony, M. 1993, ApJ, 406, 122 André, P., Motte, F., \& Bacmann, A. 1999, ApJ, 513, L57

André, P., Ward-Thompson, D., \& Barsony, M. 2000, Protostars and Planets IV, 59

Baraffe, I., Chabrier, G., Allard, F., \& Hauschildt, P. H. 1998, A\&A, 337, 403

Baraffe, I., Chabrier, G., Barman, T. S., et al. 2003, A\&A, 402, 701

Barrado y Navascués, D., \& Martín, E. L. 2003, AJ, 126, 2997

Barrado y Navascués, D., Stauffer, J. R., Morales-Calderón, M., et al. 2007, ApJ, 664,481

Bate, M. R., Bonnell, I. A., \& Bromm, V. 2002, MNRAS, 332, L65

Bertout, C., \& Genova, F. 2006, A\&A, 460, 499

Bontemps, S., Motte, F., Csengeri, T., \& Schneider, N. 2009, A\&A, submitted [arXiv: 0909.2315]

Bouy, H., \& Martin, E. L. 2009, A\&A, 504, 981

Bouy, H., Huélamo, N., Barrado y Navascués, D., et al. 2009, A\&A, 504, 199

Chen, H., Myers, P. C., Ladd, E. F., \& Wood, D. O. S. 1995, ApJ, 445, 377

di Francesco, J., Evans, N. J., II, Caselli, P., et al. 2007, prpl. conf., 17

Dolan, C. J., \& Mathieu, R. D. 1999, AJ, 118, 2409

Ducourant, C., Teixeira, R., Périé, J. P., et al. 2005, A\&A, 438, 769

Dunham, M. M., Crapsi, A., Evans, N. J., II, et al. 2008, ApJS, 179, 249 
Grazian, A., Fontana, A., Moscardini, L., et al. 2006, A\&A, 453, 507 Gutermuth, R. A., Bourke, T. L., Allen, L. E., et al. 2008, ApJ, 673, L151 Kenyon, S. J., \& Hartmann, L. 1995, ApJS, 101, 117

Harvey, P. M., Chapman, N., Lai, S.-P., et al. 2006, ApJ, 644, 307 Hatziminaoglou, E., Fritz, J., Franceschini, A., et al. 2008, MNRAS, 386, 1252 Jørgensen, J. K., Harvey, P. M., Evans, N. J., II, et al. 2006, ApJ, 645, 1246 Kauffmann, J., Bertoldi, F., Evans, N. J., II, the C2D Collaboration 2005, AN, 326,878

Kauffmann, J., Bertoldi, F., Bourke, T. L., et al. 2008, A\&A, 487, 993 Lada, C. J. 1987, IAUS, 115, 1

Lee, C. W., Bourke, T. L., Myers, P. C., et al. 2009, ApJ, 693, 1290

Lee, J.-E. 2007, JKAS, 40, 83

Luhman, K. L. 2006, ApJ, 645,

Luhman, K. L., Adame, L., D’Alessio, P., et al. 2005, ApJ, 635, L93

Mohanty, S., Basri, G., Jayawardhana, R., et al. 2004, ApJ, 609, 854

Moraux, E., Bouvier, J., \& Stauffer, J. R. 2001, A\&A, 367, 211

Myers, P. C., Adams, F. C., Chen, H., \& Schaff, E. 1998, ApJ, 492, 703
Nakajima, T., Oppenheimer, B. R., Kulkarni, S. R., et al. 1995, Nature, 378, 463 Ossenkopf, V., \& Henning, T. 1994, A\&A, 291, 943

Padoan, P., \& Nordlund, A. 2004, AJ, 617, 559

Phan-Bao, N., Riaz, B., Lee, C.-F., et al. 2008, ApJL, 689, 141

Rebolo, R., Zapatero-Osorio, M. R., \& Martin, E. L. 1995, Nature, 377, 129

Reipurth, B., \& Clarke, C. 2001, AJ, 122, 432

Shirley, Y. L., Claussen, M. J., Bourke, T. L., et al. 2007, ApJ, 667, 329

Surace, J. A., Shupe, D. L., Fang, F., et al. 2004, VizieR Online Data Catalog, 2255,0

Werner, M. W., Roellig, T. L., Low, F. J., et al. 2004, ApJS, 154, 1

White, R. J., Ghez, A. M., Reid, I. N., \& Schultz, G. 1999, ApJ, 520, 811

Whitworth, A. P., \& Zinnecker, H. 2004, A\&A, 427, 299823

Wilking, B. A., Lada, C. J., \& Young, E. T. 1989, ApJ, 340

Wu, J., Dunham, M. M., Evans, N. J., II, Bourke, T. L., \& Young, C. H. 2007, AJ, 133, 1560

Young, C. H., \& Evans, N. J., II 2005, ApJ, 627, 293

Young, C. H., Jørgensen, J. K., Shirley, Y. L., et al. 2004, ApJS, 154, 396 